Research Article

\title{
New Improved Inequalities Associated with Ratios of Gamma Functions
}

\author{
Jenica Cringanu \\ Department of Mathematics and Computer Science, "Dunarea de Jos" University of Galati, Domneasca No. 111, Galati, Romania \\ Correspondence should be addressed to Jenica Cringanu; jcringanu@ugal.ro
}

Received 13 May 2021; Accepted 5 August 2021; Published 22 September 2021

Academic Editor: Yongqiang Fu

Copyright ( $\odot 2021$ Jenica Cringanu. This is an open access article distributed under the Creative Commons Attribution License, which permits unrestricted use, distribution, and reproduction in any medium, provided the original work is properly cited.

In this paper, we improve the estimate for the products $\prod_{k=1}^{n}(4 k-3) / 4 k$ and $\prod_{k=1}^{n}(4 k-1) / 4 k$, obtained by Cringanu "inequalities associated with ratios of gamma functions."

\section{Introduction}

Cringanu [1] proved the following inequalities for the Wallis ratio:

$$
\begin{aligned}
\frac{a}{\sqrt[4]{n^{3}}} & \leq \frac{1 \cdot 5, \ldots,(4 n-3)}{4 \cdot 8, \ldots,(4 n)}<\frac{b}{\sqrt[4]{n^{3}}} \\
\frac{c}{\sqrt[4]{n}} & \leq \frac{3 \cdot 7, \ldots,(4 n-1)}{4 \cdot 8, \ldots,(4 n)}<\frac{d}{\sqrt[4]{n}}
\end{aligned}
$$

for all $n \geq 1$, with the best constants $a=(1 / 4)=0.25, b=$ $1 / \Gamma(1 / 4)=0.2758 \ldots, c=(3 / 4)=0.75$, and $d=1 / \Gamma(3 / 4)$ $=0.8160, \ldots$.

These inequalities are a consequence of the complete monotonicity on $(0, \infty)$ of the functions

$$
\begin{aligned}
& x \longrightarrow \ln \frac{(1 / \Gamma(1 / 4))^{4}}{x^{3}(\Gamma(x+(1 / 4)) / \Gamma(x+1) \Gamma(1 / 4))^{4}}, \\
& x \longrightarrow \ln \frac{(1 / \Gamma(3 / 4))^{4}}{x(\Gamma(x+(3 / 4)) / \Gamma(x+1) \Gamma(3 / 4))^{4}},
\end{aligned}
$$

where $\Gamma(x)=\int_{0}^{\infty} t^{x-1} e^{-x} \mathrm{~d} t$ for $x>0$ is the gamma function (see, for example, [2-5]).

Since

$$
\begin{aligned}
& \lim _{n \rightarrow \infty} \frac{1 \cdot 5, \ldots,(4 n-3)}{4 \cdot 8, \ldots,(4 n)} \sqrt[4]{n^{3}}=\frac{1}{\Gamma(1 / 4)}, \\
& \lim _{n \rightarrow \infty} \frac{3 \cdot 7, \ldots,(4 n-1)}{4 \cdot 8, \ldots,(4 n)} \sqrt[4]{n}=\frac{1}{\Gamma(3 / 4)},
\end{aligned}
$$

(see $[1])$, the following approximations as $n \longrightarrow \infty$ are true:

$$
\begin{aligned}
& \frac{1 \cdot 5, \ldots,(4 n-3)}{4 \cdot 8, \ldots,(4 n)} \approx \frac{1}{\Gamma(1 / 4) \sqrt[4]{n^{3}}} \\
& \frac{3 \cdot 7, \ldots,(4 n-1)}{4 \cdot 8, \ldots,(4 n)} \approx \frac{1}{\Gamma(3 / 4) \sqrt[4]{n}} .
\end{aligned}
$$

First, we concentrate on the approximation of $(1 \cdot 5, \ldots,(4 n-3) / 4 \cdot 8, \ldots,(4 n))$ and introduce the relative error sequence $w_{n}$ by the following relation for every integer $n \geq 1$ :

$$
\frac{1 \cdot 5, \ldots,(4 n-3)}{4 \cdot 8, \ldots,(4 n)}=\frac{1}{\Gamma(1 / 4) \sqrt[4]{n^{3}}} \exp w_{n} .
$$

A tool for measuring speed of convergence is a result by Mortici (lemma, [6]), who states that a sequence $x_{n}$ converging to zero is the fastest possible when the difference $x_{n}-x_{n+1}$ is the fastest possible. More precisely, if there exists $\lim _{n \longrightarrow \infty} n^{k}\left(x_{n}-x_{n+1}\right)=l$, then $\lim _{n \longrightarrow \infty} n^{k-1} x_{n}=(l / k-1)$. 
Recent results using this lemma have been given in [7-12]. In our case of $w_{n}$, we have

$$
w_{n}-w_{n+1}=\ln \frac{4 n+4}{4 n+1}-\frac{3}{4} \ln \frac{n+1}{n},
$$

and using the Maclaurin series of the function $w_{n}-w_{n+1}$ in the variable $(1 / n)$, we obtain

$$
w_{n}-w_{n+1}=-\frac{3}{32 n^{2}}+O\left(\frac{1}{n^{3}}\right)
$$

Then, $\quad \lim _{n \longrightarrow \infty} n^{2}\left(w_{n}-w_{n+1}\right)=-(3 / 32)$ and so $\lim _{n \rightarrow \infty} n w_{n}=-(3 / 32)$.

Consequently, the following approximation is true: $w_{n} \approx-(3 / 32 n)$ as $n \longrightarrow \infty$.

Similarly, if $(3 \cdot 7, \ldots,(4 n-1) /(4 \cdot 8, \ldots,(4 n)))$ $=(1 / \Gamma(3 / 4) \sqrt[4]{n}) \exp v_{n}, \quad$ then $v_{n}-v_{n+1}=-\left(3 / 32 n^{2}\right)+O\left(1 / n^{3}\right)$ and so the following approximation is true: $v_{n} \approx-(3 / 32 n)$ as $n \longrightarrow \infty$.

\section{The Main Results}

Theorem 1. (i) For all integer $n \geq 1$, we have

$$
\frac{1}{\Gamma(1 / 4) \sqrt[4]{n^{3}}} \exp \left\{-\frac{3}{32 n}-\frac{a}{n^{2}}\right\}<\frac{1 \cdot 5, \ldots,(4 n-3)}{4 \cdot 8, \ldots,(4 n)},
$$

with the best constant $a=(1 / 128)$.

(ii) For every $a<(1 / 128)$, there exists $n_{a} \in N, n \geq 1$, such that

$$
\frac{1 \cdot 5, \ldots,(4 n-3)}{4 \cdot 8, \ldots,(4 n)}<\frac{1}{\Gamma(1 / 4) \sqrt[4]{n^{3}}} \exp \left\{-\frac{3}{32 n}-\frac{a}{n^{2}}\right\}
$$

for all $n \geq n_{a}$.

Proof. We define the sequence $\left(a_{n}\right)$ for $n \geq 1$,

$$
a_{n}=\ln \frac{1 \cdot 5, \ldots,(4 n-3)}{4 \cdot 8, \ldots,(4 n)}+\ln \Gamma\left(\frac{1}{4}\right)+\frac{3}{4} \ln n+\frac{3}{32 n}+\frac{a}{n^{2}},
$$

$$
\begin{aligned}
f(n)= & \ln \frac{4 n+1}{4 n+4}+\frac{3}{4} \ln (n+1) \\
& -\frac{3}{4} \ln n+\frac{3}{32(n+1)}-\frac{3}{32 n}+\frac{a}{(n+1)^{2}}-\frac{a}{n^{2}} .
\end{aligned}
$$

The derivative of the function $f$ is equal to

$$
f^{\prime}(n)=\frac{P(n)}{32 n^{3}(n+1)^{3}(4 n+1)},
$$

where $P(n)=6 n^{3}(128 a-1)+3 n^{2}(320 a-1)+n(448 a+3)$ $+64 a$.

(i) If $a=(1 / 128)$, then $P(n)=(1 / 2)\left(9 n^{2}+13 n+1\right)$ $>0$, for all $n \geq 1$, and then $f$ is strictly increasing. Since $f(\infty)=0$, it follows that $f(n)<0$, for all $n \geq 1$, so that $\left(a_{n}\right)$ is strictly decreasing. The sequence $\left(a_{n}\right)$ converges to zero, and then it follows that $a_{n}>0$, for all $n \geq 1$, so that

$$
\frac{1}{\Gamma(1 / 4) \sqrt[4]{n^{3}}} \exp \left\{-\frac{3}{32 n}-\frac{1}{128 n^{2}}\right\}<\frac{1 \cdot 5, \ldots,(4 n-3)}{4 \cdot 8, \ldots,(4 n)},
$$

for all $n \geq 1$.

(ii) If $a<(1 / 128)$, then there exists $n_{a} \in N, n \geq 1$, such that $P(n)<0$ for all $n \geq n_{a}$, and then $f$ is strictly decreasing on $\left[n_{a}, \infty\right)$. Since $f(\infty)=0$, it follows that $f(n)>0$, for all $n \geq n_{a}$, so that $\left(a_{n}\right)_{n \geq n_{a}}$ is strictly increasing. The sequence $\left(a_{n}\right)$ converges to zero, and then it follows that $a_{n}<0$, for all $n \geq n_{a}$, so that

$$
\frac{1 \cdot 5, \ldots,(4 n-3)}{4 \cdot 8, \ldots,(4 n)}<\frac{1}{\Gamma(1 / 4) \sqrt[4]{n^{3}}} \exp \left\{-\frac{3}{32 n}-\frac{a}{n^{2}}\right\},
$$

for all $n \geq n_{a}$.

Now, we find the constant $n_{a}$ in some particular cases.

For example, if $a=(1 / 256)<(1 / 128)$, then $P(n)=-3 n^{3}+(3 / 4) n^{2}+(19 / 4) n+(1 / 4)<0$ for all $n \geq 2$ and so

and so $a_{n+1}-a_{n}=f(n)$, where

$$
\frac{1}{\Gamma(1 / 4) \sqrt[4]{n^{3}}} \exp \left\{-\frac{3}{32 n}-\frac{1}{128 n^{2}}\right\}<\frac{1 \cdot 5, \ldots,(4 n-3)}{4 \cdot 8, \ldots,(4 n)}<\frac{1}{\Gamma(1 / 4) \sqrt[4]{n^{3}}} \exp \left\{-\frac{3}{32 n}-\frac{1}{256 n^{2}}\right\}
$$

for all $n \geq 2$.

Let us remark that a direct calculus shows that these inequalities hold for $n=1$, and then

$$
\frac{1}{\Gamma(1 / 4) \sqrt[4]{n^{3}}} \exp \left\{-\frac{3}{32 n}-\frac{1}{128 n^{2}}\right\}<\frac{1 \cdot 5, \ldots,(4 n-3)}{4 \cdot 8, \ldots,(4 n)}<\frac{1}{\Gamma(1 / 4) \sqrt[4]{n^{3}}} \exp \left\{-\frac{3}{32 n}-\frac{1}{256 n^{2}}\right\}
$$


for all $n \geq 1$.

Now, if $a=(1 / 160)<(1 / 128)$, then $P(n)=-(6 / 5) n^{3}+$ $3 n^{2}+(29 / 5) n+(2 / 5)<0$ for all $n \geq 4$ and so

$$
\frac{1}{\Gamma(1 / 4) \sqrt[4]{n^{3}}} \exp \left\{-\frac{3}{32 n}-\frac{1}{128 n^{2}}\right\}<\frac{1 \cdot 5, \ldots,(4 n-3)}{4 \cdot 8, \ldots,(4 n)}<\frac{1}{\Gamma(1 / 4) \sqrt[4]{n^{3}}} \exp \left\{-\frac{3}{32 n}-\frac{1}{160 n^{2}}\right\}
$$

for all $n \geq 4$.

Let us remark that a direct calculus shows that these inequalities hold for $n=3$, and then

$$
\frac{1}{\Gamma(1 / 4) \sqrt[4]{n^{3}}} \exp \left\{-\frac{3}{32 n}-\frac{1}{128 n^{2}}\right\}<\frac{1 \cdot 5, \ldots,(4 n-3)}{4 \cdot 8, \ldots,(4 n)}<\frac{1}{\Gamma(1 / 4) \sqrt[4]{n^{3}}} \exp \left\{-\frac{3}{32 n}-\frac{1}{160 n^{2}}\right\}
$$

for all $n \geq 3$.

Remark 1. Let us remark that $\left(1 / 4 \sqrt[4]{n^{3}}\right)<\left(1 / \Gamma(1 / 4) \sqrt[4]{n^{3}}\right) \exp \left\{-(3 / 32 n)-\left(1 / 128 n^{2}\right)\right\}, \quad$ for all $n \geq 2$, and for $a \in(0,(1 / 128))$,

$$
\frac{1}{\Gamma(1 / 4) \sqrt[4]{n^{3}}} \exp \left\{-\frac{3}{32 n}-\frac{a}{n^{2}}\right\}<\frac{1}{\Gamma(1 / 4) \sqrt[4]{n^{3}}}, \quad \text { for all } n \geq 1,
$$

which is shown to improve inequality (1).

Theorem 2. (i) For all integer $n \geq 1$, we have

$$
\frac{1}{\Gamma(3 / 4) \sqrt[4]{n}} \exp \left\{-\frac{3}{32 n}+\frac{a}{n^{2}}\right\}<\frac{3 \cdot 7, \ldots,(4 n-1)}{4 \cdot 8, \ldots,(4 n)},
$$

with the best constant $a=(1 / 128)$.

(ii) For every $a>(1 / 128)$, there exists $n_{a} \in N, n_{a} \geq 1$, such that

$$
\frac{3 \cdot 7, \ldots,(4 n-1)}{4 \cdot 8, \ldots,(4 n)}<\frac{1}{\Gamma(3 / 4) \sqrt[4]{n}} \exp \left\{-\frac{3}{32 n}+\frac{a}{n^{2}}\right\}
$$

for all $n \geq n_{a}$.

Proof. We define the sequence $\left(a_{n}\right)$ for $n \geq 1$,

$$
a_{n}=\ln \frac{3 \cdot 7, \ldots,(4 n-1)}{4 \cdot 8, \ldots,(4 n)}+\ln \Gamma\left(\frac{3}{4}\right)+\frac{1}{4} \ln n+\frac{3}{32 n}-\frac{a}{n^{2}},
$$

and so $a_{n+1}-a_{n}=f(n)$, where

$$
\begin{aligned}
f(n)= & \ln \frac{4 n+3}{4 n+4}+\frac{1}{4} \ln (n+1) \\
& -\frac{1}{4} \ln n+\frac{3}{32(n+1)}-\frac{3}{32 n}-\frac{a}{(n+1)^{2}}+\frac{a}{n^{2}} .
\end{aligned}
$$

The derivative of the function $f$ is equal to

$$
f^{\prime}(n)=\frac{Q(n)}{32 n^{3}(n+1)^{3}(4 n+3)},
$$

where $Q(n)=6 n^{3}(1-128 a)+3 n^{2}(5-448 a)+n(9-832 a)$ $-192 a$.

(i) If $a=(1 / 128)$, then $Q(n)=(1 / 2)\left(9 n^{2}+5 n-3\right)>0$, for all $n \geq 1$, and then $f$ is strictly increasing.

Since $f(\infty)=0$, it follows that $f(n)<0$, for all $n \geq 1$, so that $\left(a_{n}\right)$ is strictly decreasing. The sequence $\left(a_{n}\right)$ converges to zero, and then it follows that $a_{n}>0$, for all $n \geq 1$, so that

$\frac{1}{\Gamma(3 / 4) \sqrt[4]{n}} \exp \left\{-\frac{3}{32 n}+\frac{1}{128 n^{2}}\right\}<\frac{3 \cdot 7, \ldots,(4 n-1)}{4 \cdot 8, \ldots,(4 n)}$,

for all $n \geq 1$.

(ii) If $a>(1 / 128)$, then there exists $n_{a} \in N, n \geq 1$, such that $Q(n)<0$ for all $n \geq n_{a}$, and then $f$ is strictly decreasing on $\left[n_{a}, \infty\right)$. Since $f(\infty)=0$, it follows that $f(n)>0$, for all $n \geq n_{a}$, so that $\left(a_{n}\right)_{n \geq n_{a}}$ is strictly increasing. The sequence $\left(a_{n}\right)$ converges to zero, and then it follows that $a_{n}<0$, for all $n \geq n_{a}$, so that

$$
\frac{3 \cdot 7, \ldots,(4 n-1)}{4 \cdot 8, \ldots,(4 n)}<\frac{1}{\Gamma(3 / 4) \sqrt[4]{n}} \exp \left\{-\frac{3}{32 n}+\frac{a}{n^{2}}\right\} \text {, }
$$

for all $n \geq n_{a}$. 
Now, we find the constant $n_{a}$ in some particular cases. for all $n \geq 1$, and so

For example, if $a=(1 / 64)>(1 / 128)$, then

$$
Q(n)=-6 n^{3}-6 n^{2}-4 n-3<0,
$$

$$
\frac{1}{\Gamma(3 / 4) \sqrt[4]{n}} \exp \left\{-\frac{3}{32 n}+\frac{1}{128 n^{2}}\right\}<\frac{3 \cdot 7, \ldots,(4 n-1)}{4 \cdot 8, \ldots,(4 n)}<\frac{1}{\Gamma(3 / 4) \sqrt[4]{n}} \exp \left\{-\frac{3}{32 n}+\frac{1}{64 n^{2}}\right\}
$$

for all $n \geq 1$.

Now, if $a=(1 / 120)>(1 / 128)$, then $Q(n)=-(1 / 15)$ $\left(6 n^{3}-57 n^{2}-31 n+24\right)<0$ for all $n \geq 10$ and so

$$
\frac{1}{\Gamma(3 / 4) \sqrt[4]{n}} \exp \left\{-\frac{3}{32 n}+\frac{1}{128 n^{2}}\right\}<\frac{1 \cdot 5, \ldots,(4 n-3)}{4 \cdot 8, \ldots,(4 n)}<\frac{1}{\Gamma(3 / 4) \sqrt[4]{n}} \exp \left\{-\frac{3}{32 n}+\frac{1}{120 n^{2}}\right\},
$$

for all $n \geq 10$.

Let us remark that a direct calculus shows that these inequalities hold for $n \in\{1,2, \ldots, 9\}$, and then

$$
\frac{1}{\Gamma(3 / 4) \sqrt[4]{n}} \exp \left\{-\frac{3}{32 n}+\frac{1}{128 n^{2}}\right\}<\frac{1 \cdot 5, \ldots,(4 n-3)}{4 \cdot 8, \ldots,(4 n)}<\frac{1}{\Gamma(3 / 4) \sqrt[4]{n}} \exp \left\{-\frac{3}{32 n}+\frac{1}{120 n^{2}}\right\}
$$

for all $n \geq 1$.

Remark 2. Let us remark that $(3 / 4 \sqrt[4]{n})<(1 / \Gamma(3 / 4) \sqrt[4]{n}) \exp \left\{-(3 / 32 n)+\left(1 / 128 n^{2}\right)\right\}$, for all $n \geq 1$, and for $a>(1 / 128)$,

$$
\frac{1}{\Gamma(3 / 4) \sqrt[4]{n}} \exp \left\{-\frac{3}{32 n}+\frac{a}{n^{2}}\right\}<\frac{1}{\Gamma(3 / 4) \sqrt[4]{n}}, \quad \text { for all } n>\frac{32 a}{3}
$$

which is shown to improve inequality (2).

\section{Data Availability}

The data used to support the findings of this study are available from the author upon request.

\section{Conflicts of Interest}

The author declares that there are no conflicts of interest.

\section{Acknowledgments}

The APC was funded by "Dunarea de Jos" University of Galati, Romania.

\section{References}

[1] J. Cringanu, "Inequalities associated with ratios of gamma functions," Bulletin of the Australian Mathematical Society, vol. 97, no. 3, pp. 453-458, 2018.
[2] M. Abramowitz and I. A. Stegun, Handbook of Mathematical Functions with Formulas, Graphs, and Mathematical Tables, Dover Publications, NY, USA, 1972.

[3] C.-P. Chen and F. Qi, "Completely monotonic function associated with the gamma functions and proof of wallis' inequality," Tamkang Journal of Mathematics, vol. 36, no. 4, pp. 303-307, 2005.

[4] C. Mortici, V. G. Cristea, and D. Lu, "Completely monotonic functions and inequalities associated to some ratio of gamma function," Applied Mathematics and Computation, vol. 240, pp. 168-174, 2014.

[5] D. Widder, The Laplace Transform, Princeton University Press, Princeton, NJ, USA, 1941.

[6] C. Mortici, "Product approximations via asymptotic integration," The American Mathematical Monthly, vol. 117, no. 5, pp. 434-441, 2010.

[7] N. Batir, "Very accurate approximations for the factorial function," Journal of Mathematical Inequalities, vol. 4, no. 3, pp. 335-344, 2010.

[8] D. Lu and X. Wang, "A generated approximation related to the gosper's formula and the ramanujan's formula," Journal of Mathematical Analysis and Applications, vol. 406, no. 1, pp. 287-292, 2013.

[9] D. Lu, "A generated approximation related to burnside's formula," Journal of Number Theory, vol. 136, pp. 414-422, 2014.

[10] C. Mortici, "A quicker convergence toward the gamma constant with the logarithm term involving the constant $e$," Carpathian Journal of Mathematics, vol. 26, no. 1, pp. 86-91, 2010.

[11] C. Mortici, "New improvements of the stirling formula," Applied Mathematics and Computation, vol. 217, no. 2, pp. 699-704, 2010.

[12] C. Mortici, "Fast convergences toward euler-mascheroni constant," Computational and Applied Mathematics, vol. 29, no. 3, pp. 479-491, 2010. 